\title{
Age-Related Changes in the Neurophysiology of Language in Adults: Relationship to Regional Cortical Thinning and White Matter Microstructure
}

\author{
Nobuko Kemmotsu, ${ }^{1,2}$ Holly M. Girard, ${ }^{2}$ N. Erkut Kucukboyaci, ${ }^{2,3}$ Linda K. McEvoy, ${ }^{2,4}$ Donald J. Hagler Jr, ${ }^{2,4}$ \\ Anders M. Dale, ${ }^{2,4,5}$ Eric Halgren, ${ }^{2,4,5}$ and Carrie R. McDonald ${ }^{1,2,3}$ \\ ${ }^{1}$ Department of Psychiatry, University of California, San Diego, La Jolla, California 92093-0603, ${ }^{2}$ Multimodal Imaging Laboratory, University of California, \\ San Diego, La Jolla, California $92037^{3}$ SDSU/UCSD Joint Doctoral Program in Clinical Psychology, San Diego, California 92120, ${ }^{4}$ Department of Radiology, \\ University of California, San Diego, San Diego, California 92103-0834, and 5 Department of Neurosciences, University of California, San Diego, La Jolla, \\ California 92093-0662
}

Although reading skill remains relatively stable with advancing age in humans, neurophysiological measures suggest potential reductions in efficiency of lexical information processing. It is unclear whether these age-related changes are secondary to decreases in regional cortical thickness and/or microstructure of fiber tracts essential to language. Magnetoencephalography, volumetric MRI, and diffusion tensor imaging were performed in 10 young ( 18 - 33 years) and 10 middle-aged (42-64 years) human individuals to evaluate the spatiotemporal dynamics and structural correlates of age-related changes in lexical-semantic processing. Increasing age was associated with reduced activity in left temporal lobe regions from 250 to $350 \mathrm{~ms}$ and in left inferior prefrontal cortex from 350 to $450 \mathrm{~ms}$ (i.e., N400). Hierarchical regression indicated that age no longer predicted left inferior prefrontal activity after cortical thickness and fractional anisotropy (FA) of the uncinate fasciculus (UF) were considered. Interestingly, FA of the UF was a stronger predictor of the N400 response than cortical thickness. Age-related reductions in left-lateralization of language responses were observed between 250 and $350 \mathrm{~ms}$, and were associated with left temporal thinning and frontotemporal FA reductions. N400 reductions were not associated with poorer task performance. Rather, increasing age was associated with reduction in the left prefrontal N400, which in turn was also associated with slower response time. These results reveal that changes in the neurophysiology of language occur by middle age and appear to be partially mediated by structural brain loss. These neurophysiological changes may reflect an adaptive process that ensues as communication between left perisylvian regions declines.

\section{Introduction}

Among human adults, age-related reductions in lexical-semantic processing have been reported across a wide variety of tasks, including tasks that require speeded lexical decisions, category judgments, and stem completions (Wlotko et al., 2010). Although general reading ability and semantic knowledge are well preserved into old age, the speed and efficiency with which lexical-semantic judgments are made appear to peak in young

Received Jan. 9, 2012; revised June 12, 2012; accepted July 10, 2012.

Author contributions: A.M.D., E.H., and C.R.M. designed research; N.K., H.M.G., N.E.K., and C.R.M. performed research; L.K.M., D.J.H., A.M.D., and E.H. contributed unpublished reagents/analytic tools; N.K., H.M.G., N.E.K., and C.R.M. analyzed data; N.K., L.K.M., and C.R.M. wrote the paper.

The work was supported by National Institutes of Health Grants K23NS056091 (C.R.M.) and R01NS065838 (C.R.M.).

E.H. has equity interest in CorTechs Labs, and also serves on its board of directors. A.M.D. is a founder and holds equity in CorTechs Labs and also serves on the Scientific Advisory Board. The terms of this arrangement have been reviewed and approved by the University of California, San Diego in accordance with its conflict of interest policies. The remaining authors declare no competing financial interests.

Correspondence should be addressed to Nobuko Kemmotsu, 8950 Villa La Jolla Dr., Suite C101, La Jolla, CA 92093-0841. E-mail: nkemmotsu@ucsd.edu.

DOI:10.1523/JNEUROSCI.0136-12.2012

Copyright $\odot 2012$ the authors $\quad 0270-6474 / 12 / 3212204-10 \$ 15.00 / 0$ adulthood and decline thereafter (Kutas and Iragui, 1998; Wlotko et al., 2010).

The effects of aging on lexicosemantic processing have been studied using a variety of functional imaging modalities, including electroencephalography (EEG), functional magnetic resonance imaging (fMRI), and more recently magnetoencephalography (MEG) (Aine et al., 2005). Electrophysiological studies have demonstrated changes in the amplitude, latency, or distribution of event-related potentials (ERPs) associated with language, with a primary focus on reduction of the N400 response (Kutas and Iragui, 1998). Similarly, fMRI studies have demonstrated reduced activation in older adults during language tasks in perisylvian regions (Cabeza et al., 2002; Logan et al., 2002). These neural changes associated with lexicalsemantic processing may result in decreased task efficiency or the recruitment of alternative networks to support language function (Wingfield and Grossman, 2006; Wlotko et al., 2010).

Aging is also associated with cortical and subcortical structural changes that could affect neurophysiological and behavioral measures of lexical-semantic processing. Compared with young adults, reduction in cortical thickness in middle-aged individuals appears prominent in heteromodal association cortex, including dorsolateral prefrontal and lateral temporal lobe regions, with 


\begin{tabular}{|c|c|c|c|c|c|}
\hline & \multicolumn{2}{|l|}{ Young } & \multicolumn{2}{|c|}{ Middle-aged } & \multirow{2}{*}{$\begin{array}{l}\text { Group difference } t \\
\text { statistics }\end{array}$} \\
\hline & Mean & SD & Mean & SD & \\
\hline Age (years) & 25.89 & 4.45 & 49.40 & 7.67 & $t=-7.60, p<0.001$ \\
\hline Education (years) & 16.57 & 1.99 & 16.00 & 2.45 & $t=0.49, p=0.63$ \\
\hline Single word reading (WTAR) & 116.75 & 8.50 & 112.78 & 13.09 & $t=0.73, p=0.48$ \\
\hline Vocabulary T-score & 65.13 & 6.96 & 65.14 & 8.97 & $t=0.00, p=1.00$ \\
\hline Category fluency SS & 13.43 & 2.57 & 13.22 & 2.99 & $t=0.15, p=0.87$ \\
\hline Letter fluency SS & 11.71 & 2.56 & 11.67 & 2.83 & $t=0.04, p=0.97$ \\
\hline $\begin{array}{l}\text { Finger tapping dominant } \\
\text { hand }\end{array}$ & 51.89 & 5.83 & 52.20 & 9.33 & $t=-0.07, p=0.94$ \\
\hline MEG task accuracy (\% correct) & 90.94 & 8.11 & 89.83 & 6.37 & $t=0.31, p=0.76$ \\
\hline MEG task RT (ms) & 697.83 & 88.08 & 754.86 & 147.64 & $t=-0.98, p=0.35$ \\
\hline MEG task RT, log transformed & 2.79 & 0.09 & 2.90 & 0.10 & $t=-2.46, p=0.03$ \\
\hline
\end{tabular}

WTAR, Wechsler Test of Adult Reading; SS, scaled score.

relative sparing of primary sensory cortex (McGinnis et al., 2011). In addition, studies have indicated that decreases in white matter integrity, as measured by fractional anisotropy (FA), occur by middle age and are most pronounced in prefrontal regions. These changes in the white matter microstructure are also observed in the association tracts that connect frontal cortex to more posterior brain regions, such as the inferior fronto-occipital fasciculus (IFOF), uncinate fasciculus (UF), and superior longitudinal fasciculus (Salat et al., 2005b; Perry et al., 2009). These studies indicate structural changes in cortical regions and white matter tracts critical to language functioning, suggesting a possible structural underpinning for the functional brain changes observed in response to linguistic tasks.

The goal of this study was to evaluate, through the use of a semantic judgment task, age-related changes in the spatiotemporal dynamics of lexical-semantic processing, and to determine whether age-related changes in the strength of language-related MEG responses are associated with regional cortical thinning and/or reductions in white matter integrity. We studied 20 individuals from 18 to 64 years of age using MEG, volumetric MRI, and diffusion tensor imaging (DTI). We predicted that the source strength of an MEG component involved in early lexical retrieval and subsequent semantic processing (i.e., N400 response) would decrease with age, and that these changes would be associated with cortical thinning in perisylvian cortex, as well as with reductions in the FA values of language-related white matter association tracts [arcuate fasciculus (AF), IFOF, and UF]. We further hypothesized that declines in the functional measures would be associated with reduced task efficiency (i.e., slower response time).

\section{Materials and Methods Participants}

Ten young (18-33 years; 6 females) and 10 middle-aged (42-64 years; 8 females) right-handed adults with no known history of neurological disorder, loss of consciousness, or serious medical or psychiatric conditions completed the study. The study was approved by the Institutional Review Board at the University of California, San Diego (UCSD) and each participant's consent was obtained in accordance with the ethical standards promulgated in the Declaration of Helsinki. Handedness in all participants was assessed with the Edinburgh Handedness Inventory (Oldfield, 1971). Table 1 provides demographics and neuropsychological data for our participant groups.

\section{Procedure}

Semantic judgment task

All participants completed a semantic judgment task in which they viewed words presented one at a time on a screen, and were instructed to respond by lifting a finger in response to low-frequency target items (i.e., names of animals). Task stimuli were presented visually as white letters on a black background in Arial font at $<5^{\circ}$ visual angle. Stimuli consisted of 300 novel words (NWs) presented only once, 300 "old" words (30 repetitions of 10 words), 300 false font (FF) stimuli, and 90 target words (i.e., animals). For the purpose of this paper, only the NW and FF conditions were examined. All NW stimuli were 4-8 letter nouns, with a written lexical frequency of 3-80 per 10 million (Francis and Kucera, 1982). The FF stimuli comprised alphabet-like characters matched in size and number of characters to each NW to control for visual features of the stimuli (McDonald et al., 2009). The experimental task was organized into two separate lists, each consisting of 15 blocks, with $\sim 40$ stimuli per block. NW and FF stimuli were presented in random order with $\sim 10$ of each per block. Stimuli were presented for $900 \mathrm{~ms}$ with a stimulus onset asynchrony (SOA) that varied randomly between 1200 and $1700 \mathrm{~ms}$ (average SOA, $1450 \mathrm{~ms}$ ). The sequence of stimulus conditions was balanced to ensure that each condition was preceded by every other condition with equal likelihood. The tasks were programmed using Presentation software (Neurobehavioral Systems).

\section{MEG acquisition}

Magnetic fields were recorded by an Elekta-Neuromag whole-head MEG system (Elekta) with 204 planar DC-SQUID (direct current superconducting quantum interference device) gradiometers and 102 magnetometers in a magnetically shielded room (Imedco) at the UCSD Radiology Imaging Laboratory. During recording, subjects sat upright in the dewar and were instructed to remain still and minimize blinking. EOG electrodes placed below and above the eye were used to detect eye blinks and movements. Horizontal EOG electrodes were placed lateral to the eyes to detect saccades. The translation between the MEG coordinate systems and each participant's structural MRI was made using four head position coils placed on the scalp and fiducial landmarks that were digitized to allow for later registration with MR images (Hamalainen et al., 1993). Signals were recorded continuously with $1000 \mathrm{~Hz}$ sampling rate and minimal online filtering $(0.1-200 \mathrm{~Hz})$. Data were then low-pass filtered off line at $20 \mathrm{~Hz}$ (transition band, $4 \mathrm{~Hz}$ ), high-pass filtered at $0.2 \mathrm{~Hz}$ (transition band, $0.4 \mathrm{~Hz}$ ), linearly detrended, baseline corrected using a $200 \mathrm{~ms}$ prestimulus baseline, and downsampled by a factor of four before separate averages were created for each subject. Single trials were rejected based on eye blinks (amplitudes exceeding $300 \mu \mathrm{V}$ in the EOG electrode) and large amplitude signals in MEG channels. Automated rejection based on amplitude criteria was supplemented by visual inspection. Data were inspected off line to ensure that saccades were not time-locked to the components of interest. Following visual inspection and artifact rejection, each subject's NW and FF responses [i.e., event-related fields; (ERFs)] were created by averaging across the two runs.

\section{MRI acquisition}

Magnetic resonance imaging was performed on a General Electric $1.5 \mathrm{~T}$ Excite HD scanner with an eight-channel phased-array head coil. Image acquisitions included a conventional three-plane localizer, GE calibration scan, two T1-weighted 3D structural scans (TE, $3.8 \mathrm{~ms}$; TR, $10.7 \mathrm{~ms}$; flip angle, $8^{\circ}$; bandwidth, $31.25 \mathrm{~Hz} /$ pixel; FOV, $25.6 \mathrm{~cm}$; matrix, $192 \times$ 256; slice thickness, $1.0 \mathrm{~mm}$ ), and a diffusion-weighted (DW) sequence. Diffusion data were acquired using single-shot echo-planar imaging with isotropic $2.5 \mathrm{~mm}$ voxels (matrix size, $96 \times 96$; FOV , $24 \mathrm{~cm}$; 47 axial slices; slice thickness, $2.5 \mathrm{~mm}$; partial $k$-space acquisition; TE, $75.6 \mathrm{~ms}$; TR, $12.3 \mathrm{~s}$ ), covering the entire cerebrum and brainstem without gaps. One volume series was acquired with 51 diffusion gradient directions using a $b$ value of $1000 \mathrm{~mm}^{2} / \mathrm{s}$ with an additional $b=0$ volume. For use in nonlinear $\mathrm{B} 0$ distortion correction, two additional $b=0$ volumes were acquired with either forward or reverse phase-encode polarity. Image files in DICOM (Digital Imaging and Communications in Medicine) format were transferred to a Linux workstation for further processing using an in-house, automated processing stream written with Matlab (Mathworks).

Structural MRI preprocessing. Two T1-weighted images were rigidly registered to each other, averaged, and reoriented into a common space, similar to alignment based on the anterior commissure-posterior com- 
missure line. The two T1-weighted images were averaged together to increase the contrast to noise of the images for the purpose of improving cortical thickness estimates. Images were corrected for nonlinear warping caused by nonuniform fields created by the gradient coils (Jovicich et al., 2006). Image intensities were corrected for spatial sensitivity inhomogeneities in the eight-channel head coil by normalizing with the ratio of a body coil scan to a head coil scan.

Diffusion image preprocessing. Six preprocessing steps were performed: (1) head motion between scans was removed by rigid body registration between the $b=0$ images of each DW scan; (2) within-scan motion was removed by calculating diffusion tensors, synthesizing of DW volumes from those tensors, and rigid body registering each data volume to its corresponding synthesized volume; (3) image distortion in the DW volumes caused by eddy currents was minimized by nonlinear optimization; (4) image distortion caused by magnetic susceptibility artifacts was minimized with a nonlinear B0-unwarping method using paired images with opposite phase-encode polarities (Chang and Fitzpatrick, 1992; Morgan et al., 2004; Reinsberg et al., 2005); (5) images were also corrected for gradient nonlinearities; and (6) images were resampled using cubic interpolation to $1.875 \mathrm{~mm}^{3}$ isotropic voxels.

\section{MEG source analysis}

To estimate the neural generators of the observed MEG signals and to determine time courses of activity in different cortical regions, distributed source estimates were calculated from gradiometer data using dynamic statistical parametric mapping (dSPM) (Dale et al., 2000). This method is based on the assumption that the main generators of MEG and EEG signals are localized in the gray matter. Once the exact shape of the cortical surface is known, this information can be used to reduce the MEG solution space. Furthermore, normalization procedures are used that take into account the noise sensitivity at each spatial location, allowing for statistical parametric maps that provide information about the estimated signal at each location relative to the noise. First, the cortical surface was subsampled to approximately 2500 dipole locations per hemisphere (Dale et al., 2000). Second, the forward solution at each location was calculated using boundary element method (Oostendorp and Van Oosterom, 1992). Third, dipole power was estimated at each cortical location every $4 \mathrm{~ms}$ and divided by the predicted noise power obtained from all conditions for each individual. This method generates statistical maps for each condition of interest that are F-squared distributed and represent the signal-to-noise ratio at each dipole location across the cortical surface. The square roots of these values were then averaged on the cortical surface across individuals after aligning their sulcal-gyral patterns (Fischl et al., 1999b; Dale and Halgren, 2001). Cortical activity maps for the NW and the FF conditions were generated for each participant. The subtraction of FF from NW was used to estimate language processing because it minimizes the contribution of activity associated with non-language-related functions (e.g., sensory and attentional processing) that would presumably also be evoked by false fonts.

Time windows were selected based on previous MEG (Dhond et al., 2001; Marinkovic et al., 2003) and intracranial (Halgren et al., 1994, 2006) studies of lexicosemantic processing that have revealed the approximate time course associated with visual word-form processing $(\sim 140-$ $170 \mathrm{~ms})$, initial linking of a word form to meaning ( $\sim 250 \mathrm{~ms})$, and peak lexical-semantic processing $(\sim 350-450 \mathrm{~ms} ; \mathrm{N} 400)$. Although the two later time windows reflect overlapping and extended processes, activation has been shown to peak earlier in temporal lobe regions than in prefrontal regions (Tse et al., 2007). Therefore, the responses are analyzed separately in this study to better highlight these spatiotemporal effects. Visual responses were also extracted between 80 and $120 \mathrm{~ms}$ to evaluate the effects of aging on early sensory processing. Individual subject and group data were inspected to confirm that the selected time windows were broad enough to capture the ERFs of interest in both groups. To reduce the number of comparisons, analyses were only performed for ROIs that were of theoretical interest within each time window. Thus, ROI analyses were performed using regions implicated in early sensory (pericalcarine), visual word-form (fusiform), and lexicalsemantic (temporal pole, middle temporal, superior temporal, and infe- rior prefrontal) processing of written text, and the average time courses were extracted from these selected ROIs.

\section{Surface reconstruction and cortical thickness estimates}

T1-weighted images were used to construct models of each participant's cortical surfaces using FreeSurfer software 4.5.0 (http://surfer.nmr.mgh. harvard.edu). From this reconstructed surface, measures of cortical thickness were obtained using the procedure described by Fischl and Dale (2000). Sulcal and gyral features across individual participants were aligned by morphing each participant's brain to an average spherical representation that allows for accurate matching of cortical thickness measurement locations among participants, while minimizing metric distortion (Fischl et al., 1999a). To improve signal-to-noise ratio, thickness estimates were smoothed on the average surface using a $15 \mathrm{~mm}$ full width at half maximum Gaussian kernel.

Cortical thickness estimates were computed at each vertex across the cortical mantle and within gyral-based ROIs that were described by Desikan et al. (2006). Mean thickness for each ROI was calculated by averaging the cortical thickness measurements based on the unsmoothed data at each vertex within a given ROI. In this study, these ROI measures were further combined and averaged to produce lobar (frontal, lateral temporal, medial temporal, parietal, and occipital) ROIs.

\section{White matter fiber tract segmentation and calculations}

Fiber tract FA values were obtained using a probabilistic diffusion tensor atlas that was developed using in-house software written in Matlab and $\mathrm{C}++$. A full description of the atlas and the steps used to create the atlas are described by Hagler et al. (2009).

For each participant, T1-weighted images were used to nonlinearly register the brain to a common space, and diffusion tensor orientation estimates were compared with the atlas to obtain a map of the relative probability that a voxel belongs to a particular fiber given the location and similarity of diffusion orientations. Voxels identified with FreeSurfer's automated brain segmentation (Fischl et al., 2002) as CSF or gray matter were excluded from the fiber ROIs. Average FA was calculated for each fiber ROI, weighted by fiber probability, so that voxels with low probability of belonging to a given fiber contributed minimally to average FA values. In the current study, this probabilistic atlas-based method was used to obtain average FA values for the right and left UF, AF, and IFOF due to their proposed importance for lexical-semantic processing (Mandonnet et al., 2007; Friederici, 2009; Martino et al., 2010) (Fig. 1).

\section{Statistical analysis}

MEG analysis

For the ROI analyses, each participant's NW responses were normalized to the mean of the subject's FF response between 80 and $450 \mathrm{~ms}$. This normalization was performed to control for nonlanguage and extrinsic variables that could contribute to group differences (e.g., head size or position affecting distance from the dewar). First, to test for group differences in early sensory processing, we performed $t$ tests on the latency and amplitude of the visual response, defined as the first peak identified in each individual between 70 and $120 \mathrm{~ms}$ after word onset in a pericalcarine ROI. Peaks in the waveforms were detected using Eli Billauer's peakdet (http://www.billauer.co.il/peakdet.html). This algorithm searches for minima and maxima that are a minimum difference from surrounding maxima or minima, respectively. The minimum difference chosen was 1 (arbitrary units of dSPM $\vee F)$. Peak latency and amplitude values were obtained for the NW responses and averaged across left and right hemisphere pericalcarine ROIs.

To test for group differences in visual word-form processing, a repeated measures ANOVA (RMANOVA) was performed on the average source strength within the fusiform ROI from 140 to $200 \mathrm{~ms}$ with hemisphere as a within-subject factor. To examine group differences in lexical-semantic processing, an omnibus RMANOVA was performed on the average source strengths during the $250-350 \mathrm{~ms}$ and $350-450 \mathrm{~ms}$ time windows within four frontotemporal ROIs (temporal pole, middle temporal gyrus, superior temporal gyrus, and inferior prefrontal). Pearson correlation analysis was also performed to examine the linear relationship between age-related and language-related sources. 

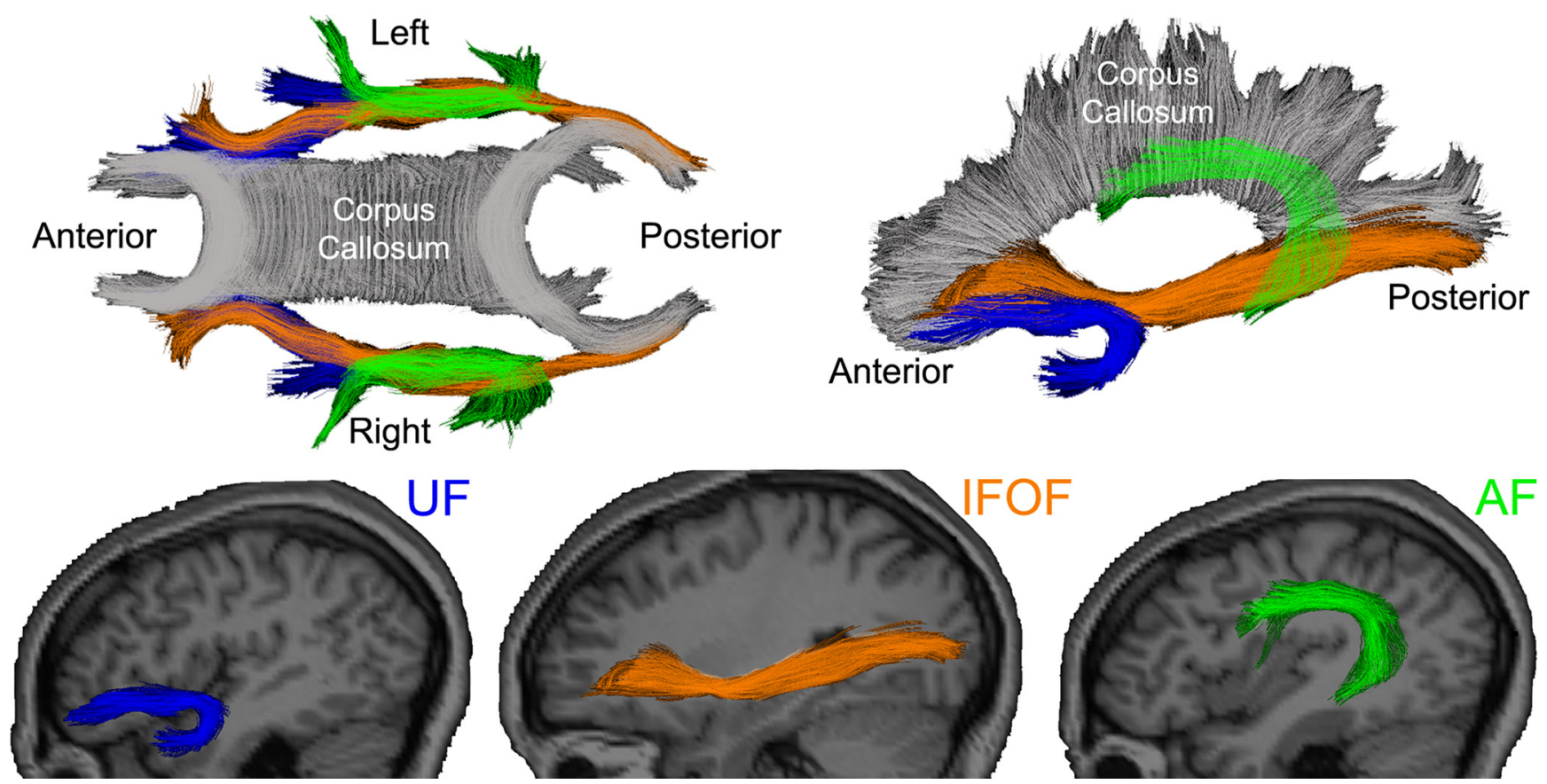

Figure 1. Sagittal views of the selected fiber tracts. Individual fiber tracts are shown projected on their corresponding T1-weighted images using Tractoview software. Color-coding is included to assist with identification of the fibers in the superimposed images.

Cortical thickness/DTI analysis

Pearson correlations were performed to examine the relationship between age and regional cortical thickness and between age and fiber tract FA values.

\section{MEG-structural regression analysis}

To examine the extent to which regional cortical thickness and fiber tract FA explained variability in the source strength of the N400 response, hierarchical regression analyses were performed. For examining the contribution of cortical thickness to N400 source strength, the average cortical thickness value of an ROI was entered at step 1 and then age was entered at step 2 . This analysis was only justified and performed when age showed a significant correlation with cortical thickness and with N400 source strength in a given ROI. A separate hierarchical regression was performed to examine the possible contribution of fiber tract FA to agerelated changes in N400 source strengths. When both cortical thickness and fiber FA contributed to N400 source strengths, hierarchical regression was performed with those two variables at step 1, followed by an addition of age at step 2, to investigate relative contribution of cortical thickness and fiber FA values.

\section{Results}

Table 1 presents the characteristics of the participants in the current study and their performance on neuropsychological tests and the MEG semantic judgment task. The young and the middle-aged groups were comparable in years of education attained and in the scores achieved on measures of general cognitive functioning and language. Response time (RT) and accuracy of the MEG task performance were available for 17 of 20 participants. Accuracy is reported as the total percentage correct. The mean difference in RTs and the log-transformed RTs are also reported. The latter score was used in the analysis because the raw RT produced a non-Gaussian distribution that was not well suited for parametric analysis (Whelan, 2008). Mean accuracy did not differ between the young and middle-aged groups, whereas middle-aged individuals were slower to respond than young adults $(t=-2.46, p=0.03)$.

\section{MEG analysis}

Figure 2 portrays the cortical activity maps of the NW minus FF responses for the young and middle-aged groups for the time windows of interest. The mean cortical activity maps of the subtraction reveal minimal activity from 80 to $120 \mathrm{~ms}$, indicating that the NW and FF conditions were well matched for visual stimulus features. This is followed by activity in bilateral fusiform and lateral occipital cortex from $\sim 140$ to $170 \mathrm{~ms}$. The fusiform response appears stronger in the left hemisphere of the younger group, a finding consistent with the role of the left fusiform in visual word-form processing (Cohen et al., 2002; Vigneau et al., 2005; Seghier and Price, 2011). By $250 \mathrm{~ms}$, the patterns between the groups diverge. From 250 to $350 \mathrm{~ms}$, the young group shows a strongly left-lateralized pattern of activity in lateral and inferior temporal and inferior frontal cortex that is sustained throughout the 350-450 ms time window. Conversely, the middle-aged group shows a more bilateral pattern of activity in lateral temporal cortex at $\sim 250-350 \mathrm{~ms}$. In addition, source strength appears much reduced from 350 to $450 \mathrm{~ms}$ in the middle-aged group, with an attenuated response in inferior frontal cortex. Figure 3 shows the time course of the NW and FF waveforms for each group, extracted from key ROIs. These waveforms provide additional support for the strong and sustained left-lateralized frontotemporal responses to NWs in the young group that are more bilateral (and perhaps right-lateralized) in the middle-aged group. This difference is particularly reflected in the late (350$450 \mathrm{~ms}$ ) left inferior prefrontal response that appears attenuated in the middle-aged group.

$t$ tests performed within the pericalcarine ROI revealed a significant group difference in latency $\left(t_{(18)}=-2.16, p=0.045\right)$ but not in amplitude $\left(t_{(18)}=-1.05, p=0.31\right)$ of the early visual response. On average, the visual response of the young adults peaked $\sim 10$ ms earlier than that of the middle-aged group (mean latency, $86.8 \mathrm{~ms}$ vs $96.9 \mathrm{~ms}$ ). Analysis of source strengths within the fusiform ROI from 140 to $170 \mathrm{~ms}$, thought to reflect word- 

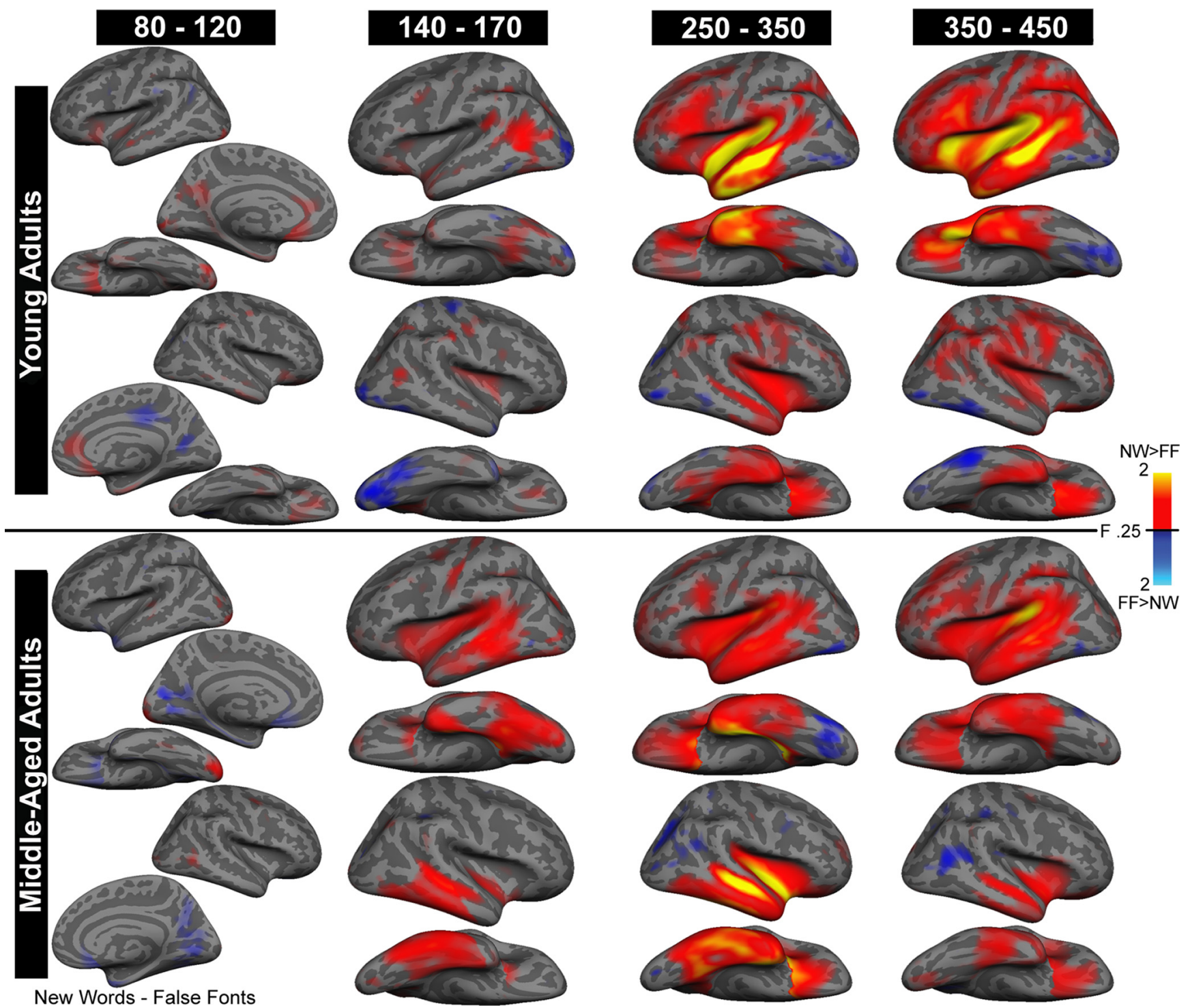

New Words - False Fonts

Figure 2. Average dSPMs of cortical responses to NW minus FF for the young and middle-aged adults for four time windows of interest. Activity is displayed on the inflated lateral and ventral surface of each hemisphere. The medial view is also shown for the early latency $(80-120 \mathrm{~ms})$ response. Sulci and gyri are shown in dark and light gray, respectively. Color bars range from minimum (red) to maximum (yellow) normalized dipole strength and represent signal-to-noise estimates that follow a square root of $F$ distribution. Cool color areas represent areas of FF greater than NW condition estimates and warm color areas represent NW greater than FF condition estimates.

form processing, revealed a significant main effect of hemisphere $\left(F_{(1,18)}=6.57, p=0.02\right)$ supporting the left-lateralized response observed in the surface maps and waveforms for both groups, but no main effect of group, or interaction of group by hemisphere.

Table 2 presents the means and SDs of the normalized NW source strengths. The omnibus RMANOVA in the 250-350 ms time window with ROI and hemisphere as within subject factors revealed a significant group by hemisphere interaction $\left(F_{(1,18)}=\right.$ $10.83, p=0.004)$. Follow-up tests indicated that in the left hemisphere, but not in the right hemisphere, young adults showed significantly greater source strengths than the middle-aged adults $\left(F_{(1,18)}=10.75, p=0.004\right)$ during this time window. Furthermore, the young group showed significantly greater source strengths in the left hemisphere relative to the right $\left(F_{(1,9)}=\right.$ $31.64, p<0.001)$ while middle-aged group did not show a significant hemispheric difference $\left(F_{(1,9)}=0.01, p=0.94\right)$.

To further explore the age-related differences in laterality of source strengths within the 250-350 ms time window, a laterality index (LI) was calculated for each ROI according to the following formula: $(L-R) /[(L+R) / 2]$, where $L$ and $R$ indicate left- and right-sided source strengths, respectively. Positive values of this index indicate leftward asymmetry, while negative values indicate rightward asymmetry. Correlational analysis demonstrated that responses to NWs became less lateralized as age increased in the temporal pole $(r=-0.485, p=0.030)$, middle temporal $(r=-0.694, p=0.001)$, and superior temporal $(r=$ $-0.541, p=0.014)$ ROIs. LIs were not significantly associated with task accuracy or speed.

The omnibus RMANOVA in the 350-450 ms time window (i.e., the peak $\mathrm{N} 400$ response) showed a significant main effect of $\operatorname{ROI}\left(F_{(3,54)}=16.89, p<0.001\right)$, hemisphere $\left(F_{(1,18)}=13.23, p=\right.$ $0.002)$, and group $\left(F_{(1,18)}=4.79, p=0.042\right)$. This indicates that activity was left-lateralized in both groups, and young adults showed greater activity overall relative to the middle-aged group. Unlike the 250-350 ms time window, there was no significant interaction of group by hemisphere. Analyses of correlation be- 


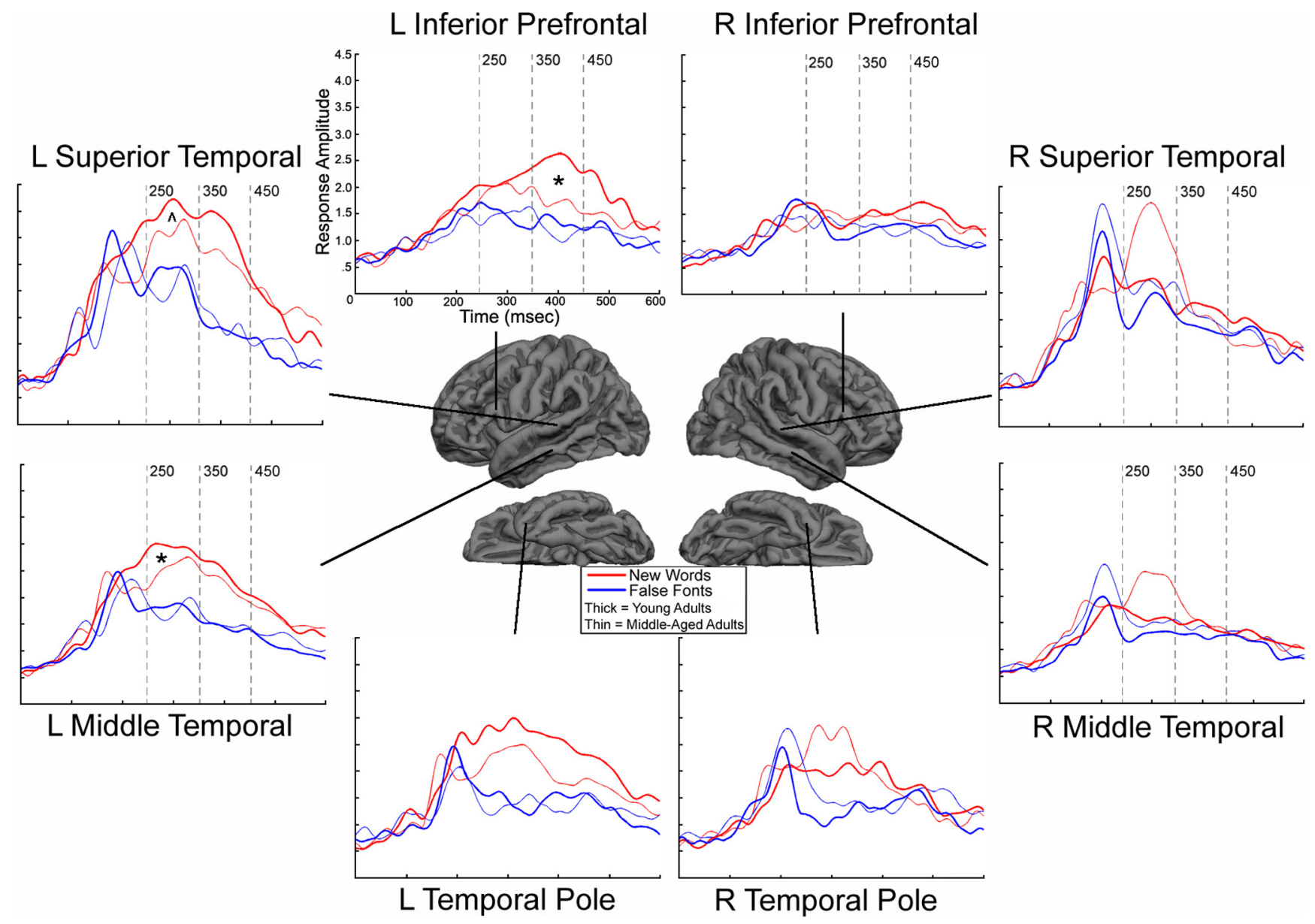

Figure 3. MEG NW waveforms (red) and FF waveforms (blue) for the young (thick lines) and middle-age (thin lines) groups from 0 to 600 ms within key ROIs.y-axis values reflect noise-normalized dipole strengths within an ROI. Asterisks denote time windows where group differences were detected in the normalized NW waveform analysis. Caret $(\wedge)$ denotes a trend. L, left; R, right.

Table 2. Means and SDs of the normalized source strengths in response to novel words for the four ROls at $250-350 \mathrm{~ms}$ and $350-450 \mathrm{~ms}$ time windows

\begin{tabular}{|c|c|c|c|c|c|c|c|c|}
\hline & \multicolumn{4}{|c|}{ Left hemisphere } & \multicolumn{4}{|c|}{ Right hemisphere } \\
\hline & \multicolumn{2}{|c|}{ Young } & \multicolumn{2}{|c|}{ Middle-aged } & \multicolumn{2}{|c|}{ Young } & \multicolumn{2}{|c|}{ Middle-aged } \\
\hline & Mean & SD & Mean & SD & Mean & SD & Mean & SD \\
\hline \multicolumn{9}{|l|}{$250-350 \mathrm{~ms}$} \\
\hline Temporal pole & 1.60 & 0.43 & 1.28 & 0.39 & 1.15 & 0.37 & 1.39 & 0.54 \\
\hline Middle temporal & 1.68 & 0.27 & 1.34 & 0.31 & 0.94 & 0.16 & 1.24 & 0.43 \\
\hline Superior temporal & 2.22 & 0.62 & 1.75 & 0.57 & 1.50 & 0.39 & 1.93 & 0.91 \\
\hline Inferior prefrontal & 1.27 & 0.32 & 1.04 & 0.33 & 0.82 & 0.21 & 0.81 & 0.28 \\
\hline \multicolumn{9}{|l|}{$350-450 \mathrm{~ms}$} \\
\hline Temporal pole & 1.52 & 0.70 & 1.11 & 0.52 & 1.11 & 0.43 & 1.05 & 0.38 \\
\hline Middle temporal & 1.45 & 0.43 & 1.27 & 0.35 & 0.86 & 0.21 & 0.91 & 0.20 \\
\hline Superior temporal & 2.13 & 0.90 & 1.73 & 0.51 & 1.26 & 0.28 & 1.28 & 0.48 \\
\hline Inferior prefrontal & 1.50 & 0.49 & 0.97 & 0.40 & 0.85 & 0.33 & 0.81 & 0.23 \\
\hline
\end{tabular}

tween age and source strength of each ROI indicated that left inferior prefrontal region was the only ROI that showed statistically significant correlation with age in this time window (Table $3)$. In the left inferior prefrontal ROI, RT was significantly correlated with $\mathrm{N} 400$ source strength $(r=-0.51, p=0.036)$.

The omnibus RMANOVAs were repeated with a subset of the sample $(n=17)$, after excluding the three participants for whom behavioral MEG data were unavailable. The pattern of our findings remained the same, with the significant group by hemisphere interaction at $250-350 \mathrm{~ms}$ time window $\left(F_{(1,15)}=7.87, p=\right.$
0.013), and the significant main effect of group in the 350-450 ms time window $\left(F_{(1,15)}=4.96, p=0.042\right)$. Thus, neither the MEG source strengths nor the lateralization effects appeared to be significantly influenced by the three participants without MEG behavioral data.

\section{Cortical thickness and DTI analyses}

Figure 4 displays maps of the association between cortical thickness and age at each vertex on the cortical surface. These maps reveal age-related thinning that is most apparent in prefrontal and cingulate cortex bilaterally. Correlations at the lobar level support prominent age-related thinning in frontal $(r=-0.67$, $p<0.001)$ and cingulate $(r=-0.81, p<0.001)$ cortices, but also reveal modest thinning in the lateral temporal $(r=-0.49, p=$ $0.028)$ and parietal cortex $(r=-0.54, p=0.013)$. With respect to fiber tract FA, increasing age was associated with decreasing FA values in the bilateral IFOF, left UF, and left AF (Table 4).

\section{Regression analyses}

Given the age-related differences in the source strength of the MEG N400 response, cortical thickness, and fiber tract FA values, hierarchical regression analyses were performed to investigate the contribution of regional thinning and FA decreases to age-related source strength reductions. Among the temporal and frontal ROIs, only the left inferior prefrontal ROI met conditions for the hierarchical analysis. That is, only this region showed a significant correlation between age and cortical thickness and a significant 
Table 3. Correlation coefficients among age, source strengths, and cortical thickness at $250-350 \mathrm{~ms}$ and $350-450 \mathrm{~ms}$ in the frontotemporal R0ls

\begin{tabular}{|c|c|c|c|c|c|c|c|c|c|c|}
\hline \multirow[b]{3}{*}{ ROls } & \multicolumn{5}{|c|}{ Left hemisphere } & \multicolumn{5}{|c|}{ Right hemisphere } \\
\hline & \multirow[b]{2}{*}{ Age and CT } & \multicolumn{2}{|l|}{$250-350 \mathrm{~ms}$} & \multicolumn{2}{|l|}{$350-450 \mathrm{~ms}$} & \multirow[b]{2}{*}{ Age and CT } & \multicolumn{2}{|l|}{$250-350 \mathrm{~ms}$} & \multicolumn{2}{|l|}{$350-450 \mathrm{~ms}$} \\
\hline & & Age and Amp & Amp and $\mathrm{CT}$ & Age and Amp & Amp and $\mathrm{CT}$ & & Age and Amp & Amp and CT & Age and Amp & Amp and $\mathrm{CT}$ \\
\hline Temporal pole & -0.29 & -0.22 & 0.26 & -0.30 & $0.61^{b}$ & -0.16 & $0.40^{c}$ & -0.30 & 0.09 & -0.43 \\
\hline Middle temporal & $-0.50^{b}$ & $-0.50^{b}$ & $0.43^{c}$ & -0.20 & 0.44 & -0.32 & $0.41^{c}$ & -0.03 & 0.10 & -0.38 \\
\hline Superior temporal & $-0.56^{a}$ & -0.37 & $0.45^{b}$ & -0.20 & 0.35 & $-0.56^{a}$ & 0.37 & -0.30 & -0.08 & 0.13 \\
\hline Inferior prefrontal & $-0.49^{b}$ & -0.28 & 0.16 & $-0.55^{b}$ & 0.42 & $-0.61^{a}$ & 0.07 & -0.10 & -0.02 & -0.00 \\
\hline
\end{tabular}

CT, cortical thickness; Amp, amplitude of the MEG response.

${ }^{a} p<0.01$.

${ }^{b} p<0.05$.

$c_{p}<0.10$ (trend).

correlation between age and N400 source strength (Table 3). For fiber tracts, FA value of the left UF correlated with peak N400 source strength of the inferior prefrontal ROI and with age (Table 4). Given these results, hierarchical regression analyses were performed on the source strength of the 350-450 ms response within the left inferior prefrontal ROI, with age, cortical thickness, and UF FA values as predictors. Age alone accounted for $30.1 \%$ of the amount of variance of the left inferior prefrontal N400 source strength $(p=0.012)$. When cortical thickness of the left inferior prefrontal ROI was entered at step 1, the amount of variance explained by age was no longer significant $(15.7 \%, p=0.062)$. Similarly, when FA value of the UF was entered at step 1 , the amount of variance accounted for by age was no longer significant $(7.5 \%$, $p=0.169)$. When both cortical thickness and UF FA values were entered at step 1, regression coefficient of the UF FA was significant ( $p=0.038$ ) but not that of the inferior prefrontal cortical thickness $(p=$ 0.252 ), indicating that the unique contribution of FA was more meaningful (partial correlation coefficient, 0.478 ) in predicting left prefrontal source strength than that of cortical thickness (partial correlation coefficient, 0.276 ) or age.

Given the age effect on reduced later-

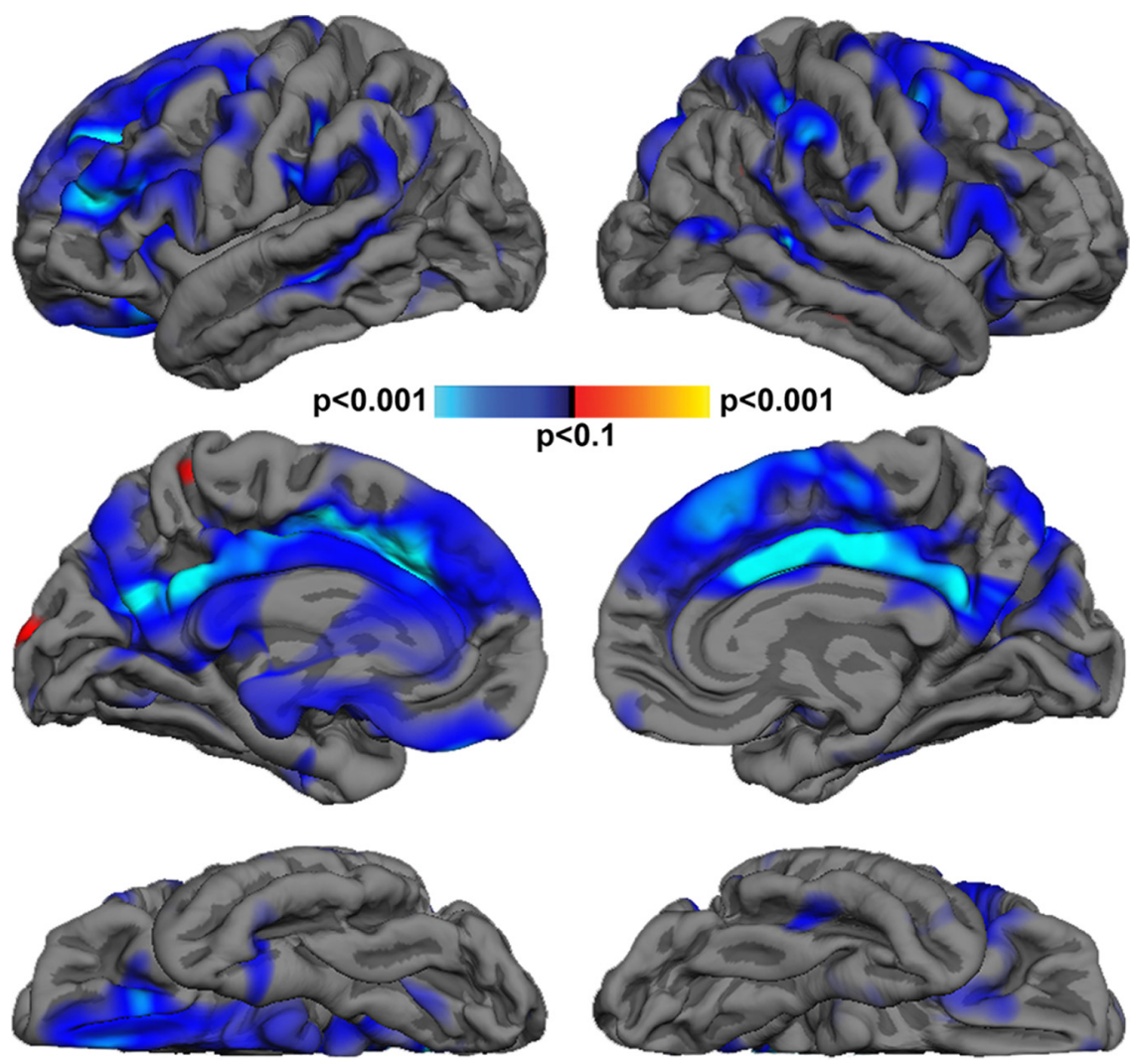

Figure 4. Cortical thickness maps demonstrating the linear association between age and thickness across the cortical surface. Blue indicates areas where increasing age was associated with thinning, while red indicates areas where increasing age was associated with increased cortical thickness. Significance thresholds range from $p<0.01$ (dark blue) to $p<0.0001$ (cyan).

ality in the 250-350 ms window, we also explored the relationship between FA values of the fiber tracts, the local cortical thickness, and LI of the source strengths of the middle temporal ROI. Middle temporal LI was positively correlated with cortical thickness of the left middle temporal ROI. This means that, as the thickness of the ROI decreased, response within the ROI became less lateralized. Right hemisphere thickness of the ROI did not show significant relationship with LI. Bilateral IFOF and left UF were positively correlated with middle temporal LI. Neither cortical thickness nor white matter FA values mediated the effect of age on the LIs: age continued to explain a significant amount of variance even with the structural values in the model (Table 5).

\section{Discussion}

The current study investigated (1) the effects of advancing age on MEG activity associated with lexical-semantic processing, and

(2) the extent to which age-related differences in source strength and laterality could be explained by regional cortical thinning and changes in white matter microstructure. To our knowledge, this is the first MEG study that has examined age-related changes in lexical-semantic processing as a function of regional cortical thinning and fiber tract changes. Our primary MEG findings indicate that age did not significantly affect the strength of responses related to early visual processing and word-form identification in the pericalcarine and fusiform regions, respectively. In contrast, age-related changes emerged in perisylvian regions during time windows previously associated with linking a word form to meaning ( $\sim 250 \mathrm{~ms}$ ) and subsequent semantic processing (i.e., N400, peaking between 350 and $450 \mathrm{~ms}$ ) (Lau et al., 2008). These changes were characterized by a reduction in the strength of the left temporal lobe responses from 250 to $350 \mathrm{~ms}$, and a reduced degree of lateralization of this response in the middle-aged com- 
Table 4. Correlation coefficients between age and $\mathrm{N} 400 \mathrm{ROI}$ source strengths, age and fiber tract $\mathrm{FAs}$, and $\mathrm{N} 400$ source strengths and FAs

\begin{tabular}{|c|c|c|c|c|c|c|c|}
\hline \multirow[b]{3}{*}{ ROls } & \multirow[b]{3}{*}{ Age } & \multicolumn{6}{|c|}{ FA values } \\
\hline & & \multicolumn{3}{|c|}{ Left hemisphere } & \multicolumn{3}{|c|}{ Right hemisphere } \\
\hline & & IFOF & UF & $A F$ & IFOF & UF & $\mathrm{AF}$ \\
\hline & & $-0.61^{a}$ & $-0.59^{a}$ & $-0.46^{b}$ & $-69^{a}$ & -0.22 & $-0.41^{\mathrm{c}}$ \\
\hline Left temporal pole & -0.30 & 0.05 & 0.37 & -0.18 & -0.00 & -0.13 & -0.19 \\
\hline Left middle temporal & -0.20 & 0.25 & $0.51^{a}$ & 0.04 & 0.20 & 0.15 & -0.28 \\
\hline Left superior temporal & -0.20 & 0.21 & $0.50^{b}$ & 0.12 & 0.29 & 0.33 & -0.11 \\
\hline Left inferior prefrontal & $-0.55^{b}$ & $0.39^{c}$ & $0.56^{b}$ & 0.32 & $0.40^{c}$ & 0.18 & 0.32 \\
\hline Right temporal pole & 0.09 & -0.11 & -0.22 & 0.06 & -0.13 & -0.12 & 0.15 \\
\hline Right middle temporal & 0.10 & -0.17 & -0.31 & -0.04 & -0.11 & -0.17 & 0.07 \\
\hline Right superior temporal & -0.08 & -0.19 & -0.28 & -0.22 & -0.09 & 0.01 & 0.19 \\
\hline Right inferior prefrontal & -0.02 & 0.03 & -0.19 & 0.17 & 0.05 & 0.17 & $0.47^{b}$ \\
\hline
\end{tabular}

Table 5. Amount of variance accounted for by cortical thickness, fiber tract FAs, and age in the middle temporal laterality indices

\begin{tabular}{lllll}
\hline & $R^{2}$ & $p$ & $\Delta R^{2}$ by age & $p$ \\
\hline Left middle temporal cortical thickness & $41.5 \%$ & 0.002 & $18.4 \%$ & 0.012 \\
Left IFOF & $33.1 \%$ & 0.005 & $16.8 \%$ & 0.024 \\
Right IFOF & $30.6 \%$ & 0.007 & $16.0 \%$ & 0.032 \\
Left UF & $30.5 \%$ & 0.007 & $18.7 \%$ & 0.019 \\
\hline
\end{tabular}

$R^{2}$, amount of variance accounted for by each of the structural variables entered at step 1 of the hierarchical regression; $\Delta R^{2}$, amount of additional variance accounted for by age at step 2 of the hierarchical regression.

pared with the young adults. This was followed by decreased activity in middle-aged adults from 350 to $450 \mathrm{~ms}$ (i.e., peak N400 response) observed in left inferior prefrontal cortex and associated with a longer RT. These data suggest age-related changes in perisylvian responses by middle age, during the later stages of language processing in left temporal and prefrontal regions associated with controlled retrieval and selection of lexical representations (Lau et al., 2008). The observed age-related reduction in the MEG response is consistent with prior ERP literature that identified reductions in N400 effects apparent by middle age (Harbin et al., 1984; Gunter et al., 1992; Kutas and Iragui, 1998; Federmeier et al., 2003). We extend this literature by providing a more detailed account of the specific spatial and temporal characteristics of the language-related reductions observed in young to middle-age adults.

Second, we demonstrate that regional cortical thinning and reductions in white matter FA values occur by middle age. Cortical thinning was most apparent in prefrontal and cingulate regions, with modest reductions in temporoparietal cortex. Early thinning in prefrontal cortex is consistent with the hypothesis that late-maturing areas of the brain are particularly vulnerable to age-related atrophy by middle age (Davis et al., 2009; Fjell et al., 2009; McGinnis et al., 2011). Furthermore, age-related changes in frontotemporal white matter microstructure have been demonstrated by middle age (Salat et al., 2005a; Davis et al., 2009). Our findings support these data and provide a possible cortical and subcortical substrate for N400 reductions in prefrontal cortex.

The most novel findings in this study were that age-related prefrontal thinning and loss of white matter microstructure of the UF mediated the effects of age on N400 source strengths in left inferior prefrontal cortex, and that the FA values of the left UF was a stronger predictor of N400 source strength than was local cortical thinning. The significant correlation between UF FA and the $\mathrm{N} 400$ source strength in the left inferior prefrontal region may reflect the UF's strong connections between temporal lobe regions involved in linking a word form to meaning and inferior prefrontal regions implicated in subsequent semantic processing (Anwander et al., 2007; Frey et al., 2008; Friederici, 2009). That is, the UF connects the temporal pole to inferior prefrontal cortex and has been previously implicated in language functions, including lexical retrieval and semantic associations (Papagno, 2011). Recent ERP studies have shown relationships between the magnitude of the brain's electrophysiological response and white matter microstructure (Westlye et al., 2009; Colrain et al., 2011). Our data strengthen the view that the microstructure of white matter fiber tracts subserving the N400 generators may affect the nature of the $\mathrm{N} 400$ response, perhaps by facilitating efficient neuronal conduction (Peters, 2009; Roberts et al., 2009). P300 latencies have been found to be associated more with white matter connectivity than with the gray matter volume (Cardenas et al., 2005), and our results indicate that similar relationships may hold for the strength of N400 response. The UF and surrounding white matter may play a critical role in the strength of the N400 response by providing a framework for communication between temporal and prefrontal perisylvian generators.

Although previous research has suggested a link between cortical thickness and the magnitude of brain responses, there is evidence that cortical-functional relationships vary with age. Fjell et al. (2007) found that cortical thickness was related to P300 amplitudes in elderly but not in young adults. We observed a reliable relationship between age and cortical thickness, but relationships between cortical thickness and MEG source strengths were more limited (Tables 3, 4). Gautam et al. (2011) found associations between cortical thickness and cognitive performance in individuals aged 64-68 years, but not in those aged 44-48 years, suggesting that different structural-function relationships exist at different ages. It may be that white matter FA value is a good predictor of N400 strength from young to middle adulthood, while cortical thinning becomes a predictor later in life. Our results suggest that the effects of age on the N400 response is complex and mediated by multiple factors, of which white matter microstructure may play a more central role than cortical thinning in middle age.

We observed an age-related reduction in lateralization during the 250-350 $\mathrm{ms}$ in the temporal lobe ROIs in the context of comparable levels of task accuracy between the two age groups. Decreased language lateralization has been shown with fMRI in individuals 18-40 years of age during a semantic decision task (Springer et al., 1999), and this change in laterality was indepen- 
dent of task performance. Szaflarski et al. (2006) also found that language lateralization in the lateral temporal and frontal area decreased with age in a sample of adults ranging from 25 to 70 years old. Our study adds to the existing literature by providing a specific spatiotemporal profile of reductions in language lateralization with age. It is noteworthy that post hoc correlation analyses at the individual ROIs revealed strong trends for older individuals to demonstrate larger responses in the right temporal pole and middle temporal ROIs (Table 3 ). These data suggest that reductions in left hemisphere language activity may be accompanied by slight increases in homologous right hemisphere regions-a finding that previous studies suggested may represent a compensatory response associated with aging (Cabeza et al., 2002, 2004).

Although task accuracy did not differ between the young and middle-aged groups in our study, middle-aged individuals showed prolonged RT associated with a decrease in the left inferior prefrontal $\mathrm{N} 400$ response. This finding provides evidence for a behavioral correlate of age-related N400 reductions that may represent decreased efficiency in the controlled aspects of lexicalsemantic processing (Gold et al., 2009). Nevertheless, the functional significance of N400 reductions is still debated. In particular, studies of semantic priming have shown that decreased N400 responses reflect increased processing efficiency (Matsumoto et al., 2005) and fMRI studies have shown that BOLD reductions are associated with improved task performance (Vogt et al., 2007). Therefore, while there is considerable agreement that the $\mathrm{N} 400$ decreases with aging and this decrease is often observed together with slower RTs (Gunter et al., 1992; Kutas and Iragui, 1998; Federmeier et al., 2003; Wlotko et al., 2010), researchers are divided about whether age-related N400 reductions reflect increased or decreased efficiency in lexicalsemantic processing. Interpretations differ across studies. Although our study is not positioned to directly resolve this issue, we provide evidence that subtle cortical and subcortical atrophy within perisylvian regions contribute to N400 reductions, as well as to changes in the distribution of lexical-semantic responses, and that these effects occur by middle age.

Despite the theoretical value of our findings, several limitations of this study should be addressed. First, our study is limited by its relatively small sample size and restricted age range. Studying a large cohort from young to old age would likely unveil additional and/or different predictors of changes in the N400 response. Nevertheless, age effects in the N400 response even in the restricted age range examined here suggest that changes in the brain's response to a simple semantic judgment task occur relatively early in the adult aging process. Second, we chose to focus on low-frequency oscillations often associated with N400 effects (i.e., below $20 \mathrm{~Hz}$ ). However, it is possible that other interesting age-related language effects would have been captured if we had performed a spectral analysis of data in the upper $\beta$ and $\gamma$ band (Lachaux et al., 2007; McDonald et al., 2010). Last, this study examined correlations among age, MEG responses, and brain structure, and correlation analyses cannot determine causation.

In conclusion, the current study reveals changes in the neurophysiology of language that are apparent by middle age and appear to be associated with atrophy to perisylvian cortex and the underlying white matter tracts. Furthermore, we demonstrate that frontotemporal white matter compromise is a stronger predictor of N400 reductions than is cortical thinning. These results provide new insights into the neuroanatomical substrates underlying age-related changes in neurophysiology, and highlight the importance of communication between frontotemporal perisylvian regions in the strength of the N400 response.

\section{References}

Aine CJ, Adair JC, Knoefel JE, Hudson D, Qualls C, Kovacevic S, Woodruff CC, Cobb W, Padilla D, Lee RR, Stephen JM (2005) Temporal dynamics of age-related differences in auditory incidental verbal learning. Brain Res Cogn Brain Res 24:1-18.

Anwander A, Tittgemeyer M, von Cramon DY, Friederici AD, Knösche TR (2007) Connectivity-based parcellation of Broca's area. Cereb Cortex $17: 816-825$.

Cabeza R, Anderson ND, Locantore JK, McIntosh AR (2002) Aging gracefully: compensatory brain activity in high-performing older adults. Neuroimage 17:1394-1402.

Cabeza R, Daselaar SM, Dolcos F, Prince SE, Budde M, Nyberg L (2004) Task-independent and task-specific age effects on brain activity during working memory, visual attention and episodic retrieval. Cereb Cortex 14:364-375.

Cardenas VA, Chao LL, Blumenfeld R, Song E, Meyerhoff DJ, Weiner MW, Studholme C (2005) Using automated morphometry to detect associations between ERP latency and structural brain MRI in normal adults. Hum Brain Mapp 25:317-327.

Chang H, Fitzpatrick JM (1992) A technique for accurate magnetic resonance imaging in the presence of field inhomogeneities. IEEE Trans Med Imaging 11:319-329.

Cohen L, Lehéricy S, Chochon F, Lemer C, Rivaud S, Dehaene S (2002) Language-specific tuning of visual cortex? Functional properties of the visual word form area. Brain 125:1054-1069.

Colrain IM, Sullivan EV, Ford JM, Mathalon DH, McPherson SL, Roach BJ, Crowley KE, Pfefferbaum A (2011) Frontally mediated inhibitory processing and white matter microstructure: age and alcoholism effects. Psychopharmacology (Berl) 213:669-679.

Dale AM, Halgren E (2001) Spatiotemporal mapping of brain activity by integration of multiple imaging modalities. Curr Opin Neurobiol 11:202-208.

Dale AM, Liu AK, Fischl BR, Buckner RL, Belliveau JW, Lewine JD, Halgren E (2000) Dynamic statistical parametric mapping: combining fMRI and MEG for high-resolution imaging of cortical activity. Neuron 26:55-67.

Davis SW, Dennis NA, Buchler NG, White LE, Madden DJ, Cabeza R (2009) Assessing the effects of age on long white matter tracts using diffusion tensor tractography. Neuroimage 46:530-541.

Desikan RS, Ségonne F, Fischl B, Quinn BT, Dickerson BC, Blacker D, Buckner RL, Dale AM, Maguire RP, Hyman BT, Albert MS, Killiany RJ (2006) An automated labeling system for subdividing the human cerebral cortex on MRI scans into gyral based regions of interest. Neuroimage 31:968-980.

Dhond RP, Buckner RL, Dale AM, Marinkovic K, Halgren E (2001) Spatiotemporal maps of brain activity underlying word generation and their modification during repetition priming. J Neurosci 21:3564-3571.

Federmeier KD, Van Petten C, Schwartz TJ, Kutas M (2003) Sounds, words, sentences: age-related changes across levels of language processing. Psychol Aging 18:858-872.

Fischl B, Dale AM (2000) Measuring the thickness of the human cerebral cortex from magnetic resonance images. Proc Natl Acad Sci U S A 97:11050-11055.

Fischl B, Sereno MI, Dale AM (1999a) Cortical surface-based analysis. II: inflation, flattening, and a surface-based coordinate system. Neuroimage 9:195-207.

Fischl B, Sereno MI, Tootell RB, Dale AM (1999b) High-resolution intersubject averaging and a coordinate system for the cortical surface. Hum Brain Mapp 8:272-284.

Fischl B, Salat DH, Busa E, Albert M, Dieterich M, Haselgrove C, van der Kouwe A, Killiany R, Kennedy D, Klaveness S, Montillo A, Makris N, Rosen B, Dale AM (2002) Whole brain segmentation: automated labeling of neuroanatomical structures in the human brain. Neuron 33:341-355.

Fjell AM, Walhovd KB, Fischl B, Reinvang I (2007) Cognitive function, P3a/ $\mathrm{P} 3 \mathrm{~b}$ brain potentials, and cortical thickness in aging. Hum Brain Mapp 28:1098-1116.

Fjell AM, Westlye LT, Amlien I, Espeseth T, Reinvang I, Raz N, Agartz I, Salat DH, Greve DN, Fischl B, Dale AM, Walhovd KB (2009) High consis- 
tency of regional cortical thinning in aging across multiple samples. Cereb Cortex 19:2001-2012.

Francis WN, Kucera H (1982) Frequency analysis of English usage: lexicon and grammar. Boston: Houghton Mifflin.

Frey S, Campbell JS, Pike GB, Petrides M (2008) Dissociating the human language pathways with high angular resolution diffusion fiber tractography. J Neurosci 28:11435-11444.

Friederici AD (2009) Pathways to language: fiber tracts in the human brain. Trends Cogn Sci 13:175-181.

Gautam P, Cherbuin N, Sachdev PS, Wen W, Anstey KJ (2011) Relationships between cognitive function and frontal grey matter volumes and thickness in middle aged and early old-aged adults: the PATH Through Life Study. Neuroimage 55:845-855.

Gold BT, Andersen AH, Jicha GA, Smith CD (2009) Aging influences the neural correlates of lexical decision but not automatic semantic priming. Cereb Cortex 19:2671-2679.

Gunter TC, Jackson JL, Mulder G (1992) An electrophysiological study of semantic processing in young and middle-aged academics. Psychophysiology 29:38-54.

Hagler DJ Jr, Ahmadi ME, Kuperman J, Holland D, McDonald CR, Halgren E, Dale AM (2009) Automated white-matter tractography using a probabilistic diffusion tensor atlas: Application to temporal lobe epilepsy. Hum Brain Mapp 30:1535-1547.

Halgren E, Baudena P, Heit G, Clarke JM, Marinkovic K, Clarke M (1994) Spatio-temporal stages in face and word processing. I. Depth-recorded potentials in the human occipital, temporal and parietal lobes [corrected]. J Physiol Paris 88:1-50.

Halgren E, Wang C, Schomer DL, Knake S, Marinkovic K, Wu J, Ulbert I (2006) Processing stages underlying word recognition in the anteroventral temporal lobe. Neuroimage 30:1401-1413.

Hamalainen M, Hari R, Ilmoniemi RJ, Knuutila J, Lounasmaa OV (1993) Magnetoencephalography-theory, instrumentation, and applications to noninvasive studies of the working human brain. Rev Mod Phys 65:413-497.

Harbin TJ, Marsh GR, Harvey MT (1984) Differences in the late components of the event-related potential due to age and to semantic and nonsemantic tasks. Electroencephalogr Clin Neurophysiol 59:489-496.

Jovicich J, Czanner S, Greve D, Haley E, van der Kouwe A, Gollub R, Kennedy D, Schmitt F, Brown G, Macfall J, Fischl B, Dale A (2006) Reliability in multi-site structural MRI studies: effects of gradient non-linearity correction on phantom and human data. Neuroimage 30:436-443.

Kutas M, Iragui V (1998) The N400 in a semantic categorization task across 6 decades. Electroencephalogr Clin Neurophysiol 108:456-471.

Lachaux JP, Fonlupt P, Kahane P, Minotti L, Hoffmann D, Bertrand O, Baciu M (2007) Relationship between task-related gamma oscillations and BOLD signal: new insights from combined fMRI and intracranial EEG. Hum Brain Mapp 28:1368-1375.

Lau EF, Phillips C, Poeppel D (2008) A cortical network for semantics: (de) constructing the N400. Nat Rev Neurosci 9:920-933.

Logan JM, Sanders AL, Snyder AZ, Morris JC, Buckner RL (2002) Underrecruitment and nonselective recruitment: dissociable neural mechanisms associated with aging. Neuron 33:827-840.

Mandonnet E, Nouet A, Gatignol P, Capelle L, Duffau H (2007) Does the left inferior longitudinal fasciculus play a role in language? A brain stimulation study. Brain 130:623-629.

Marinkovic K, Dhond RP, Dale AM, Glessner M, Carr V, Halgren E (2003) Spatiotemporal dynamics of modality-specific and supramodal word processing. Neuron 38:487-497.

Martino J, Brogna C, Robles SG, Vergani F, Duffau H (2010) Anatomic dissection of the inferior fronto-occipital fasciculus revisited in the lights of brain stimulation data. Cortex 46:691-699.

Matsumoto A, Iidaka T, Haneda K, Okada T, Sadato N (2005) Linking semantic priming effect in functional MRI and event-related potentials. Neuroimage 24:624-634.

McDonald CR, Thesen T, Hagler DJ Jr, Carlson C, Devinksy O, Kuzniecky R, Barr W, Gharapetian L, Trongnetrpunya A, Dale AM, Halgren E (2009) Distributed source modeling of language with magnetoencephalography: application to patients with intractable epilepsy. Epilepsia 50:2256-2266.

McDonald CR, Thesen T, Carlson C, Blumberg M, Girard HM, Trongnetrpu- nya A, Sherfey JS, Devinsky O, Kuzniecky R, Dolye WK, Cash SS, Leonard MK, Hagler DJ Jr, Dale AM, Halgren E (2010) Multimodal imaging of repetition priming: Using $\mathrm{AMRI}, \mathrm{MEG}$, and intracranial EEG to reveal spatiotemporal profiles of word processing. Neuroimage 53:707-717.

McGinnis SM, Brickhouse M, Pascual B, Dickerson BC (2011) Age-related changes in the thickness of cortical zones in humans. Brain Topogr 24:279-291.

Morgan PS, Bowtell RW, McIntyre DJ, Worthington BS (2004) Correction of spatial distortion in EPI due to inhomogeneous static magnetic fields using the reversed gradient method. J Magn Reson Imaging 19:499-507.

Oldfield RC (1971) The assessment and analysis of handedness: the Edinburgh inventory. Neuropsychologia 9:97-113.

Oostendorp TF, Van Oosterom A (1992) Source parameter estimation using realistic geometry in bioelectricity and biomagnetism. In: Biomagnetic localization and 3D modeling (Nenonen J, Rajala HM, Katila T, eds). Helsinki: Helsinki University of Technology, Report TKK-F-A689.

Papagno C (2011) Naming and the role of the uncinate fasciculus in language function. Curr Neurol Neurosci Rep 11:553-559.

Perry ME, McDonald CR, Hagler DJ Jr, Gharapetian L, Kuperman JM, Koyama AK, Dale AM, McEvoy LK (2009) White matter tracts associated with set-shifting in healthy aging. Neuropsychologia 47:2835-2842.

Peters A (2009) The effects of normal aging on myelinated nerve fibers in monkey central nervous system. Front Neuroanat 3:11.

Reinsberg SA, Doran SJ, Charles-Edwards EM, Leach MO (2005) A complete distortion correction for MR images: II. Rectification of static-field inhomogeneities by similarity-based profile mapping. Phys Med Biol 50:2651-2661.

Roberts TP, Khan SY, Blaskey L, Dell J, Levy SE, Zarnow DM, Edgar JC (2009) Developmental correlation of diffusion anisotropy with auditoryevoked response. Neuroreport 20:1586-1591.

Salat DH, Tuch DS, Greve DN, van der Kouwe AJ, Hevelone ND, Zaleta AK, Rosen BR, Fischl B, Corkin S, Rosas HD, Dale AM (2005a) Age-related alterations in white matter microstructure measured by diffusion tensor imaging. Neurobiol Aging 26:1215-1227.

Salat DH, Tuch DS, Hevelone ND, Fischl B, Corkin S, Rosas HD, Dale AM (2005b) Age-related changes in prefrontal white matter measured by diffusion tensor imaging. Ann N Y Acad Sci 1064:37-49.

Seghier ML, Price CJ (2011) Explaining left lateralization for words in the ventral occipitotemporal cortex. J Neurosci 31:14745-14753.

Springer JA, Binder JR, Hammeke TA, Swanson SJ, Frost JA, Bellgowan PS, Brewer CC, Perry HM, Morris GL, Mueller WM (1999) Language dominance in neurologically normal and epilepsy subjects: a functional MRI study. Brain 122:2033-2046.

Szaflarski JP, Holland SK, Schmithorst VJ, Byars AW (2006) fMRI study of language lateralization in children and adults. Hum Brain Mapp 27:202-212.

Tse CY, Lee CL, Sullivan J, Garnsey SM, Dell GS, Fabiani M, Gratton G (2007) Imaging cortical dynamics of language processing with the eventrelated optical signal. Proc Natl Acad Sci U S A 104:17157-17162.

Vigneau M, Jobard G, Mazoyer B, Tzourio-Mazoyer N (2005) Word and non-word reading: what role for the visual word form area? Neuroimage 27:694-705.

Vogt S, Buccino G, Wohlschläger AM, Canessa N, Shah NJ, Zilles K, Eickhoff SB, Freund HJ, Rizzolatti G, Fink GR (2007) Prefrontal involvement in imitation learning of hand actions: effects of practice and expertise. Neuroimage 37:1371-1383.

Westlye LT, Walhovd KB, Bjørnerud A, Due-Tønnessen P, Fjell AM (2009) Error-related negativity is mediated by fractional anisotropy in the posterior cingulate gyrus-a study combining diffusion tensor imaging and electrophysiology in healthy adults. Cereb Cortex 19:293-304.

Whelan R (2008) Effective analysis of reaction time data. Psychol Rec $58: 475-482$.

Wingfield A, Grossman M (2006) Language and the aging brain: patterns of neural compensation revealed by functional brain imaging. J Neurophysiol 96:2830-2839.

Wlotko EW, Lee CL, Federmeier KD (2010) Language of the aging brain: event-related potential studies of comprehension in older adults. Lang Linguist Compass 4:623-638. 